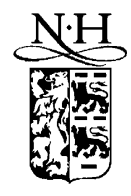

\title{
A superconducting detector endstation for high-resolution energy-dispersive SR-XRF
}

\author{
S. Friedrich ${ }^{\mathrm{a}, \mathrm{b}, *}$, T. Niedermayr ${ }^{\mathrm{a}}$, O. Drury ${ }^{\mathrm{a}}$, M.F. Cunningham ${ }^{\mathrm{a}}$, \\ M.L. van den Berg ${ }^{\mathrm{a}}$, J.N. Ullom ${ }^{\mathrm{a}}$, A. Loshak ${ }^{\mathrm{a}}$, T. Funk ${ }^{\mathrm{b}}$, S.P. Cramer ${ }^{\mathrm{b}}$, \\ J.D. Batteux ${ }^{\mathrm{a}}$, E. See ${ }^{\mathrm{a}}$, M. Frank ${ }^{\mathrm{a}}$, S.E. Labov ${ }^{\mathrm{a}}$ \\ ${ }^{a}$ Lawrence Livermore National Laboratory, P.O. Box 808, L-418, Livermore, CA 94551, USA \\ ${ }^{\mathrm{b}}$ Lawrence Berkeley National Laboratory, MS 6-2100, Berkeley, CA 94720, USA
}

\begin{abstract}
We have built a two-stage adiabatic demagnetization refrigerator (ADR) to operate cryogenic high-resolution X-ray detectors in synchrotron-based fluorescence applications. The detector is held at the end of a $40 \mathrm{~cm}$ cold finger that extends into a UHV sample chamber. The ADR attains a base temperature below $100 \mathrm{mK}$ with about $20 \mathrm{~h}$ hold time below $400 \mathrm{mK}$, and does not require pumping on the liquid $\mathrm{He}$ bath. We will discuss cryostat design and performance. (C) 2001 Elsevier Science B.V. All rights reserved.
\end{abstract}

Keywords: Adiabatic demagnetization refrigerators; Superconducting tunnel junctions; X-ray detectors

\section{Introduction}

Cryogenic X-ray detectors have received wide attention during the last decade because they combine the high energy resolution of grating spectrometers with the broadband efficiency of semiconducting energy-dispersive detectors [1]. Cryogenic detectors for the photon energy range between 0.1 and $6 \mathrm{keV}$ fall into two groups: microcalorimeters, which offer very high energy resolution between 2 and $5 \mathrm{eV}$ FWHM at the expense of low count rate around 500 counts/s [2,3], and superconducting tunnel junctions (STJs),

\footnotetext{
*Corresponding author. Tel.: + 1-925-423-1527; fax: + 1925-424-5512.

E-mail address: friedrich1@1lnl.gov (S. Friedrich).
}

which offer somewhat poorer resolution between 2 and $13 \mathrm{eV}$ FWHM but can be operated at an order of magnitude higher count rates [4-6].

High energy resolution combined with comparably high count rate capabilities makes STJ detectors attractive for synchrotron-based X-ray fluorescence spectroscopy (SR-XRF). We have developed $\mathrm{Nb}-\mathrm{Al}-\mathrm{AlOx}-\mathrm{Al}-\mathrm{Nb}$ STJ detectors and operated them in an adiabatic demagnetization refrigerator (ADR) in SR-XRF applications [6,7]. Our STJ detectors have achieved an energy resolution between 1.7 and $8.9 \mathrm{eV}$ FWHM at $50 \mathrm{eV}-1 \mathrm{keV}$, and they have been successfully operated at count rates above 10,000 counts/s $[4,6]$.

For practical SR-XRF experiments, the STJ detector, typically with an area around 
$0.1 \times 0.1 \mathrm{~mm}^{2}$, must be placed close to the sample to acquire data with a reasonable count rate. Also, since the Al vacuum vessel of a typical ADR cryostat cannot be baked out easily, residual gases can freeze out on the STJ detector and on the thin IR blocking windows in front of it. Finally, the necessity to pump on the liquid He bath consumes both time and liquid $\mathrm{He}$. We have therefore designed a new two-stage ADR for SR-XRF where the STJ detector is held at the end of a cold finger that can be inserted into a UHV sample chamber. Here, we discuss the design and performance of this detector system.

\section{Cryostat design}

Adiabatic demagnetization is a process of magnetic cooling below a liquid He bath temperature through isothermal magnetization and adiabatic demagnetization of a paramagnetic material. Magnetization lowers the entropy of the paramagnet, and the heat of magnetization is carried into a liquid He bath through a closed heat switch. After opening the heat switch, the magnetic field is decreased sufficiently slowly to keep the entropy of the paramagnet constant, thereby lowering its temperature. ADRs are compact, reliable and easy to use.

The ADR cryostat discussed here uses a commercial $30 \mathrm{~cm}$ diameter shell with a 7.41 liquid $\mathrm{N}_{2}$ and a 9.61 liquid He tank. The liquid He tank has a cylindrical $10 \mathrm{~cm}$ cavity to accommodate the $5 \mathrm{~T}$ ADR magnet with the paramagnets and a Vanadium Permendur magnetic shield. To reduce the heat load into the liquid He tank, the the ADR magnet uses high-temperature superconducting BSCCO current leads between the liquid $\mathrm{N}_{2}$ and liquid He cooled stages.

The cryostat uses two nested stages with two different paramagnets (Fig. 1) [8,3]. The first stage is cooled to a temperature around $1 \mathrm{~K}$ by a $143 \mathrm{~g}$ single crystal of gadolinium gallium garnet $\mathrm{Gd}_{3} \mathrm{Ga}_{5} \mathrm{O}_{12}$ (GGG). This stage supports a second stage which cools to a base temperature below $0.1 \mathrm{~K}$ using a home-grown $63 \mathrm{~g}$ salt pill of paramagnetic $\mathrm{Fe}\left(\mathrm{NH}_{4}\right)\left(\mathrm{SO}_{4}\right)_{2} \times 12 \mathrm{H}_{2} 0$, commonly known as FAA for ferric ammonium alum. This

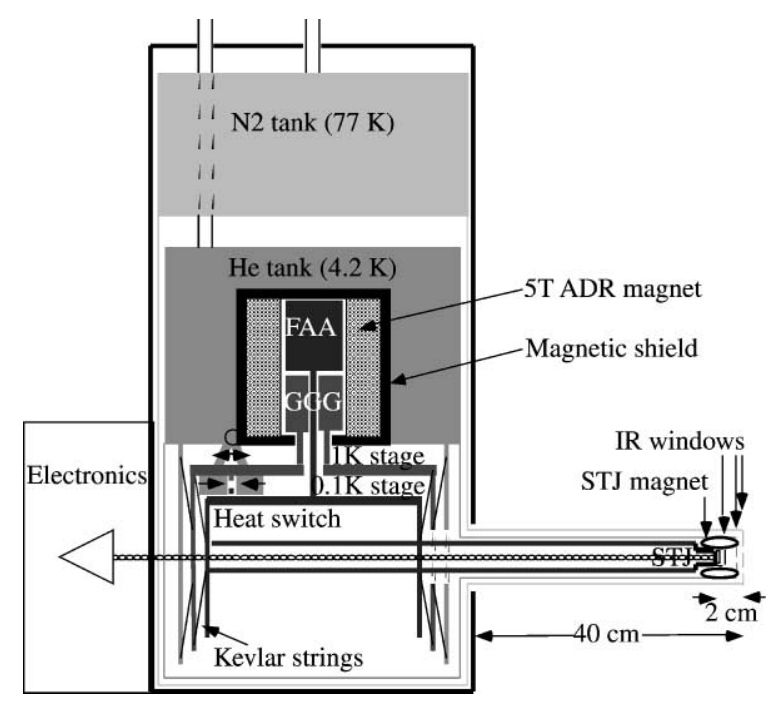

Fig. 1. Schematic cross-section of the two-stage ADR.

two-stage design allows detector operation at a base temperature below $0.1 \mathrm{~K}$ with a bath temperature of $4.2 \mathrm{~K}$ and thus does not require pumping on the liquid $\mathrm{He}$ bath. Since FAA dehydrates at temperatures above $40^{\circ} \mathrm{C}$ thereby losing its paramagnetic properties, the ADR cannot be baked out easily. We have therefore assembled the ADR in a clean room wearing latex gloves and using only ultrasonically cleaned parts and vented screws to make it as close to UHV compatible as possible.

We operate both paramagnets with a single magnet and a single heat switch. We have designed an electrically controlled mechanical heat switch to avoid air leakage at the O-ring sealed feedthroughs often found in conventional mechanical heat switches (Fig. 2). It uses an over-center-cam locking mechanism activated by $200 \mathrm{~ms} 1.5 \mathrm{~A}$ current pulses through two solenoids that toggle a stainless steel spring between two stable positions. The heat switch provides a force of $1500 \mathrm{~N}$ to each of the cold fingers. Its thermal conductance of $65 \mathrm{~mW} / \mathrm{K}$ at $4.2 \mathrm{~K}$ is limited by the thermal conductivity of the OFHC $\mathrm{Cu}$ braids between the heat switch and the He bath. After magnetization, the cold stages return to the bath temperature within $30 \mathrm{~min}$. This dominates the time required for a demagnetization cycle. 


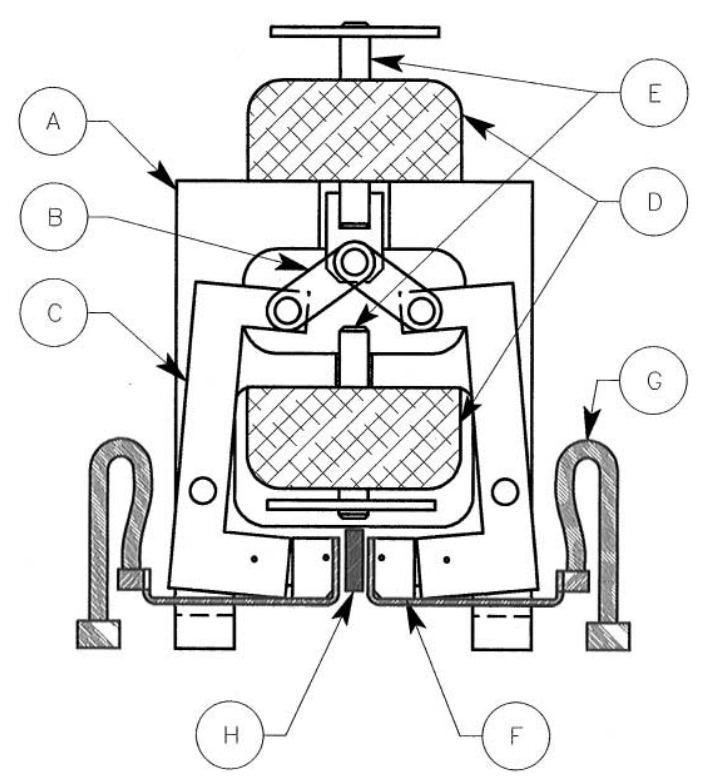

Fig. 2. Schematics of the heat switch: (A) Stainless steel (SS) frame, (B) Movable SS links to, (C) SS springs which rotate about the pivot points (circles), (D) two solenoids (crosshatched) with plungers (E) for opening (bottom) and closing (top) heat switch, (F) Au-plated OFHC $\mathrm{Cu}$ thermal links (shaded), spring-loaded to pivot at the end of the SS springs (C) for good thermal contact between the $0.1 \mathrm{~K}$ stage and $1 \mathrm{~K}$ stage fingers $(\mathrm{H})$ and the liquid $\mathrm{He}$ bath through the annealed OFHC Cu-braids $(\mathrm{G})$.

The STJ detector is mounted at the end of a Au-plated OFHC $\mathrm{Cu}$ rod. This rod is surrounded by a liquid $\mathrm{He}$ cooled Au-plated $\mathrm{OFHC} \mathrm{Cu}$ radiation shield, which also holds the STJ detector magnet needed to suppress the dc Josephson current for stable STJ operation. All of this is enclosed with a second radiation shield attached to the liquid $\mathrm{N}_{2}$ cooled stage. The entire cold finger protrudes $40 \mathrm{~cm}$ from the cryostat wall, and its diameter of $45 \mathrm{~mm}$ is sufficiently small to fit through standard gate valves. Three $500 \AA \mathrm{Al}$ on $2000 \AA$ parylene IR blocking windows on an $85 \%$ transmissive $\mathrm{Cu}$ grid at the end of the cold finger prevent room temperature radiation from heating the cold stage and causing IR induced excess noise in the detector. Their $5 \mathrm{~mm}$ diameter is determined by a trade-off between desired angle of acceptance and tolerable IR photon flux.

\section{Results and discussion}

Our two-stage ADR has attained a base temperature as low as $60 \mathrm{mK}$, which allows temperature controlled operation at temperatures down to $80 \mathrm{mK}$. The cold stage has a hold time of about $20 \mathrm{~h}$ below $400 \mathrm{mK}$, the maximum operating temperature of our STJ detectors. The liquid He hold time is about two days. The total time for a demagnetization cycle is $45 \mathrm{~min}$, yielding a duty cycle for the ADR of more than $95 \%$.

Initial X-ray fluorescence measurements at the Advanced Light Source have achieved an energy resolution between 11 and $25 \mathrm{eV}$ FWHM for photon energies between 277 and $850 \mathrm{eV}$ with $0.1 \times 0.1 \mathrm{~mm}^{2}$ STJ detectors. This is already more than sufficient to separate even weak transition metal L lines from say a strong oxygen $\mathrm{K}$ line, as shown in the emission spectrum of the metalloprotein hydrogenase (Fig. 3). On the other hand, the resolution is less than that of nominally identical STJ detectors mounted in the center of an ADR, rather than at the end of a cold finger

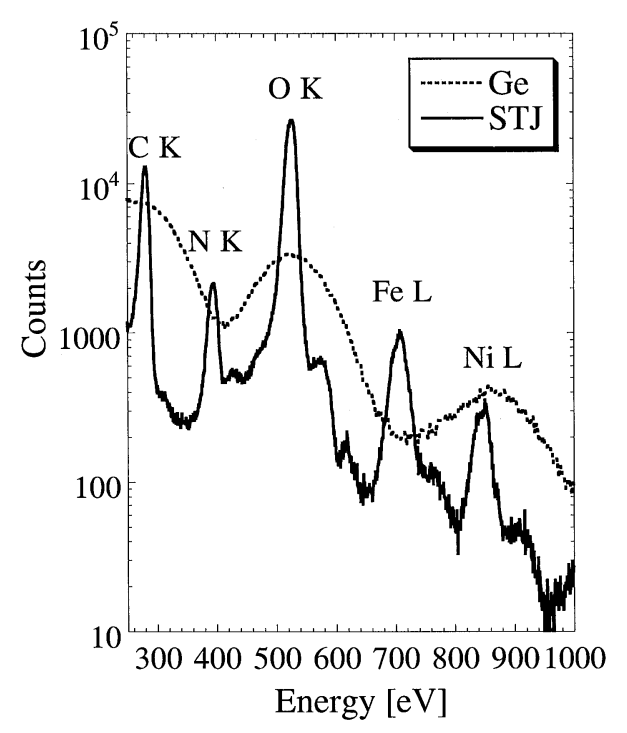

Fig. 3. Soft X-ray emission spectrum of the metalloprotein hydrogenase containing $\approx 480 \mathrm{ppm} \mathrm{Ni}$ and $\approx 5800 \mathrm{ppm} \mathrm{Fe}$ (solid line). An emission spectrum of this protein taken with a commercial 30-element Ge detector is shown for comparison. The Ni fluorescence is enhanced because of resonant excitation at the Ni L edge (dashed). 
within $2.5 \mathrm{~cm}$ of a room temperature sample $[4,6]$. This discrepancy may be due to excess noise caused by IR radiation, or due to insufficient shielding of external magnetic fields The UHV chamber vacuum was in the low $10^{-9}$ mbar range, and no signs of gas freeze-out on the IR blocking windows have been observed.

In summary, we have built a two-stage ADR with a $40 \mathrm{~cm}$ long cold finger to operate cryogenic STJ detectors in synchrotron-based X-ray fluorescence applications. It allows easy operation of STJ detectors with $\approx 10-25 \mathrm{eV} \mathrm{FWHM}$ resolution at a distance of $\approx 2.5 \mathrm{~cm}$ to the sample in a UHV chamber in the low $10^{-9}$ mbar range. This advance in detector development significantly improves the sensitivity in high-resolution soft X-ray spectroscopy of dilute samples.

\section{Acknowledgements}

This work was performed under the auspices of the US Department of Energy by University of California Lawrence Livermore National Labora- tory under Contract No. W-7405-Eng-48. Funding was provided by the NASA SADD Grant NAG 5-4137, the NASA High Energy Astrophysics SR\&T agreement W19.121, the NASA Constellation X program under S-10256G, the NIH Grant GM 44380, the DOE OBER, and the Radiometry Laboratory of the PTB, Berlin. We particularly thank R. Fliegauf, M. Veldkamp and B. Beckhoff for their efforts and feedback.

\section{References}

[1] Proceedings of the Eighth International Workshop on Low Temperature Detectors (LTD-8), Nucl. Instr. and Meth. A 444 (2000).

[2] K.D. Irwin et al., Nucl. Instr. and Meth. A 444 (2000) 184.

[3] D.A. Wollman et al., J. Microsc. 188 (1997) 196.

[4] S. Friedrich et al., IEEE Trans. Appl. Supercond. 9 (1999) 3330 .

[5] G. Angloher et al., Nucl. Instr. and Meth. A 444 (2000) 214.

[6] M. Frank et al., Rev. Sci. Instrum. 69 (1998) 25.

[7] S. Friedrich et al., J. El. Spectrosc. Relat. Phenom. 101 (1999) 891.

[8] C. Hagmann, P.L. Richards, Cryogenics 34 (1994) 221. 